\section{MS}

médecine/sciences $1.992 ; 8: 8-9$

\section{Maurice Tubiana}

\section{RÉFÉRENCES}

Correa P. The new era of cancer epidemiology. Cancer epidemiology, biomarkers. Prevention $1991 ; 1: 5-11$.

Doll R, Peto R. The causes of cancer. Quantitative estimates of avoidable risks of cancer in the United States today. I Nat Cancer Inst 1981; 66 : 1191-308.

Doll R. Are we winning the fitht against cancer? An epidemiological assessment. Eur J Cancer 1990;26:500-8.

Higginson J. Changing concepts in cancer prevention : limitations and implications for future research in environmental carcinogenesis. Cancer Res 1988 ; 48 : 1381-9.

Hill C, Benhamou E, Doyon F, Flamant R. Evolution de la mortalité par cancer en France entre 1950 et 1985. Paris : Éditions INSERM, 1986.

Muir CS. Epidemiology, basic science and the prevention of cancer. Implications for the future. Cancer Res 1990; $50: 6441-8$. Sasco AJ. Migrations et cancers. Rev Med Intern $1989 ; 10: 341-8$

\section{ADRESSE ET TIRÉS À PART}

M. Tubiana : membre de l'Institut. Académic des Sciences, 23, quai Conti, 75005 Paris, France.

\section{TIRÉS A PART}

\section{Tubiana.}

\title{
L'ÉPIDÉMIOLOGIE DU CANCER
}

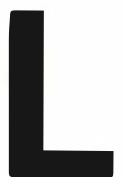

a biologie moléculaire est avec l'épidémiologie l'une des deux disciplines qui ont le plus fait progresser nos connaissances sur le cancer depuis un demi-siècle Depuis 191(), on sait provoquer des cancers chez l'animal, ce qui a permis d'identilier des agents cancérogènes, physiques ou chimiques, mais l'extrapolation de la poule ou de la souris à l'homme est aléatoire et, faute de données directes, une grande incertitude régnait sur les causes des cancers humains. Prenons l'exemple des virus. Dès 1911, P. Rous avait montré que l'injection de broyats cellulaires de certaines tumeurs provoquait, chez les animaux de même lignée, des tumeurs. Le facteur causal était donc un virus. Par des méthodes analogues, on a montré ensuite que de nombreux cancers chez des oiseaux ou des mammitères ont également à leur origine un virus. En est-il de même chez l'homme? Question restée sans réponse jusqu'au début des années 1980. L'étude, par D. Burkitt, d'un cancer atteignant les enfants dans le centre de l'Afrique montra que celui-ci était très fréquent dans les vallées, le long des cours d'eau ou sur les bords des lacs mais rare sur les hauts plateaux bien qu'il s'agisse de mêmes ethnies ayant le même mode de vie. Cela suggérait donc la responsabilité d'un virus véhiculé par un moustique. Le transport de fragments de tumeurs fraîches en Angleterre permit l'identification du virus d'Epstein-Barr (EBV), mais ce virus est apparemment banal et est trouvé che\% de nombreux enfants sains. L'enquête épidémiologique de G. de Thé montra que si l'on dose les anticorps chez des jeunes enfants en bonne santé, ce sont ceux dont le taux est le plus élevé qui ont la plus grande probabilité d'avoir un cancer. On a pu ainsi reconstituer l'histoire de la maladie: contamination massive dès les premiers mois de la vie, puis période de latence jusqu'à ce que, à l'occasion d'une baisse des défenses immunologiques provoquée par le paludisme, les cellules infestées par le virus redeviennent capables de se multiplier rapidement, des lignées de cellules précancéreuses (immortalisées) apparaissent puis, à la faveur d'une nouvelle mutation, une cellule devient pleinement cancéreuse.

Les causes des cancers humains et la prévention. Cet exemple montre que l'épidémiologie du cancer est loin d'être simplement un recensement de la fréquence des divers types de cancer. Son premier mérite est de suggérer des hypothèses sur les causes de cancers humains et d'apporter des arguments en leur faveur en liaison étroite avec les méthodes les plus modernes de la biologie. Elle permet ensuite, grâce au suivi de la fréquence des cancers, de vérifier la validité des méthodes de prévention fondées sur ces travaux.

L'exemple le plus éclatant en ce domaine, celui qui a donné à l'épidémiologie ses lettres de noblesse, est celui du tabac. Entre 1930 et 1950, on a assisté dans tous les pays occidentaux à une extraordinaire augmentation de la fréquence du cancer du poumon. Les cancérologues suspectèrent les gaz d'échappement des voitures et le bitume des routes. Il restait à en faire la preuve. Doll et Hill interrogèrent 650 malades atteints de cancer du poumon et 650 sujets témoins hospitalisés dans le même établissement pour d'autres affections. Lors du dépouillement des questionnaires, il apparut que les deux groupes ne différaient que par un seul caractère : $96 \%$ des cancéreux étaient, ou avaient été, des fumeurs contre $80 \%$ dans le groupe témoin. De plus, le nombre moven de cigarettes fumées était légèrement plus grand chez les premiers que chez les seconds. Sur ces critères, ils conclurent au rôle cancérogène du tabac, déduction qui fut accueillic avec scepticisme. Cependant, d'autres enquêtes concordantes furent rapidement publiées ; de plus, et surtout, des enquêtes prospectives, au cours desquelles plusieurs dizaines puis centaines de milliers de sujets bien portants furent interrogés puis suivis, confirmèrent le résultat. Son importance historique est grande. D'abord, il montra les conséquences néfastes d'une habitude ancienne et considérée jusque-là comme relativement peu dangereuse et ouvrit la voie d'une prévention efficace du cancer. Les optimistes crurent que la connaissance du risque de la cigarette suffirait à faire chuter sa consommation; en fait, si le public se pénétra des dangers du tabac, le pourcentage de fumeurs ne diminua que très lentement et essentiellement parmi les couches les plus instruites de la population, faisant du tabac un handicap supplémentaire des milieux les plus défavorisés. Les études épidémiologiques faites à ce propos soulignent la complexité du processus de prise de décision et l'abîme qui sépare "souhaiter " de " vouloir ". L'épidémiologie conduit ainsi à la prévention et fournit matière à des études sociologiques sur les comportements humains.

La seconde conséquence importante de la découverte de l'effet carcinogène du tabac a été de montrer le rôle des facteurs exogènes. Dans ces conditions, des milliers de nouveaux produits chimiques produits par centaines de millions de tonnes depuis la seconde révolution industrielle ne risquaient-ils pas de provoquer une augmentation de la fréquence des cancers? Depuis les années 1950, de nombreux groupes se consacrent à ces enquêtes et la création en 1965 du centre international de recherche sur le cancer fut destinée à répondre à cette préoccupation. Sans entrer dans le détail des travaux qui ont été accomplis, on peut en dégager les conclusions essentielles. Si l'on fait abstraction de l'augmentation de la fréquence des cancers dus au tabac, la fréquence des cancers à âge constant a peu varié depuis un siècle. Certains types de cancer sont aujourd'hui plus fréquents. 
Il en est ainsi, par exemple, pour le cancer du sein ou de la prostate. Inversement, d'autres cancers sont aujourd'hui moins fréquents, tels ceux de l'estomac ou du col de l'utérus. Ces variations se compensent, c'est pourquoi la fréquence globale a peu changé. Or, si l'irruption dans notre milieu de milliers de nouveaux produits chimiques avai eu un impact global notable, cette fréquence aurait dû croître une vingtaine d'années plus tard. Les deux organes qui sont les plus exposés aux pollutions sont l'estomac pour l'eau et les aliments, les poumons pour l'air Or, la fréquence des cancers de l'estomac a diminué tandis que, chez les non-fumeurs celle du cancer du poumon est restée constante.

Cependant, il faut distinguer ces études effectuécs sur des populations normalisées pour l'âge de la fréquence absolue qui, elle, a considérablement augmenté en raison de l'allongement de l'espérance de vic qui est passée de 45 ans en 1900 à 77 ans aujourd'hui. Comme l'incidence du cancer croît rapidement avec l'âge, le cancer qu causait moins de $10 \%$ de décès en 1900 en cause aujourd'hui, en France, $25 \%$. On estime que le nombre de cancers devrai croître d'environ $15 \%$ dans les 20 ans à venir pour des raisons purement démographiques. En raison des progrès de la lutte contre les affections cardio-vasculaires, le cancer doit devenir au XXI siècle la première cause de mortalité.

Les comparaisons géographiques montrent qu'à âge constant, la fréquence des différent cancers varic considérablement. Ces différences ne sont pas dues à des facteurs ethniques comme le montre la comparaison entre les Noirs vivant en Afrique ou en Amérique, ou les Japonais au Japon ou aux EtatsUnis. L'étude des populations migrantes a permis de préciser le rôle de l'environnement social et culturel. On estime aujourd'hui qu'environ $80 \%$ des cancers sont dus à cet environnement dont environ $75 \%$ liés au mode de vic individuel et $5 \%$ au milieu.

Les enquêtes épidémiologiques ont montré, pour plusicurs types de cancers, des corré lations qui, associées aux études biologiques, ont permis d'identifier les causes de ces cancers telles que certains cancérogènes chimiques (tabac, amiante, etc.) ou physique (rayons UV du soleil, rayonnements ionisants), des virus (EBV pour la maladie de Burkitt, le cancer du naso-pharynx, certaines maladies de Hodgkin, le virus de l'hépatite B pour le cancer du foic, HTLV-1 pour certaines leucémies, le virus du papillome pour les cancers du col utérin et de la verge).

Cependant, hormis les cas d'exposition massive à un cancérogène (tabac), l'origine est en général multifactorielle et un va-et-vient entre enquêtes épidémiologiques et études biologiques est nécessaire pour analyser la séquence plus ou moins complexe des phénomènes et le rôle des différents facteurs (par exemple, virus et cancérogène chimi-

$\mathrm{m} / \mathrm{s} \mathrm{n}^{\circ} \mathrm{l}$, vol. 8, janvier 92 que pour le cancer du foie, virus et infection bactérienne pour le cancer du col, excès de sel dans l'alimentation et infections bactériennes pour les cancers gastriques, etc.)

Le temps n'est plus où comme pour le tabac ou l'amiante, l'épidémiologie suffisait seule à apporter la solution. Elle reste essentielle pour apporter des données sur lesquelles s'élabore le modèle de cancérogenèse mais doit être étroitement associée à la biologie moléculaire, la virologie, les marqueurs biologiques. Pour les besoins de la prévention, il est essentiel néanmoins d'estimer les responsabilités des différents agents cancé rogènes et le bénéfice qui résulterait de leur suppression. Doll et Peto ont les premiers effectué cet exercice dans un travail resté célèbre et dont les conclusions n'ont pas été remises en question. On admet qu'en France environ $30 \%$ des morts par cancer sont dues au tabac, $10 \%$ à l'alcool, $30 \%$ à l'alimentation, $5 \%$ aux comportement sexuels ou à l'exposition aux rayons UV du soleil. Pour les $5 \%$ dus au milieu, environ $3 \%$ correspondent aux cancers professionnels, 1 à $2 \%$ à la pollution de l'air, l'eau et l'alimentation et moins de $1 \%$ à la radioactivité naturelle ou artificielle. Malgré leur caractère approximatif ces chiffres montrent les priorités : d'abord, et avan tout, lutter contre le tabac, puis mieux analyser les facteurs alimentaires nocifs (abus de matières grasses, suralimentation) ou bénéfiques (fruits et légumes riches en vitamines A, C, E), enfin consacrer les moyens nécessaires aux cancers professionnels sur le plan recherche et formation.

La pollution pose des problèmes particuliers Les données épidémiologiques montrent que son rôle est très modeste ; cependant, quelques cas où l'on a pu analyser l'influence d'une pollution massive (comme en Europe de l'Est) montrent qu'elle peut avoir un effe décelable. Pour agir de façon pertinente, il faudrait connaître la forme de la relation entre la dose et l'effet de façon à pouvoir évaluer le risque des faibles concentrations de cancérogènes auxquelles sont exposées la population à partir des risques de fortes concentrations. Malheureusement, cette relation n'est connue avec une certaine précision que pour le tabac où elle est de type $k D^{2} t^{4}$ où $\mathrm{k}$ est une constante, $\mathrm{D}$ la dose exprimée en nombre de cigarettes par jour et t le temps pendant lequel le sujet a fumé, exprimé en années.

Si ce type de relation était valable pour les autres cancérogènes, une exposition courte à de faibles concentrations, n'aurait que des conséquences minimes. Cependant, certains, pour estimer le risque, utilisent une relation linéaire où l'effet est proportionnel à la dose, quelle que soit celle-ci. Avec une telle relation, de faibles concentrations comporteraient des risques notables et c'est pourquoi certains environnementalistes américains demandent des mesures drastiques pour réduire les concentrations de polluants chimiques dans l'air et l'eau. Ces mesures coûteraient des centaines de milliards de dollars, aussi la communauté scientifique est très réticente, craignant que ces sommes gigantesques ne soient ainsi dépensées, sans bénéfice réel pour la population alors que, utilisées dans d'autres domaines, elles pourraient rendre de grands services. Ces controverses sont d'autant plus vives que les légumes et les fruits contiennent de nombreux produits naturels toxiques et cancérogènes, dont on pourrait dire, en langage finaliste, qu'ils sont destinés à éviter que les animaux n'en fassent une trop forte consommation. Or, chez l'homme, une alimentation riche en légumes et fruits, bien loin d'accroître le risque de cancer le diminue.

Le seul moyen de progresser serait d'obtenir des données directes dans le domaine des faibles doses. Plusieurs approches sont possibles: (a) enquêtes portant sur un très grand nombre de sujets. Cette méthode est très coûteuse mais constitue un ultime recours; (b) effectuer une méta-analyse regroupant plusieurs enquêtes dont chacune a fourni des différences non significatives en l'absence d'un nombre suffisant d'événements ; (c) utiliser des marqueurs biologiques sensibles, par exemple des modifications de l'ADN cellulaire (tels des adduits) dont on a établi qu'ils sont corrélés à la probabilité d'apparition d'un cancer. Il est ains concevable d'identifier des états précancéreux, beaucoup plus fréquents que les cancers.

Étant donné l'importance pratique des problèmes posés par le tabagisme passif, l'exposition à de faibles doses de rayonnements ionisants ou de toxiques chimiques, le rôle de l'alimentation dans la genèse des cancers, on peut s'attendre à une augmentation rapide de ces divers types de recherche.

Histoire naturelle des cancers. Les études épidémiologiques, nous l'avons vu à propos de la maladie de Burkitt, permettent de reconstituer l'évolution d'un cancer depuis l'apparition des lésions précancéreuses jusqu'à la période terminale. L'épidémiologie a ainsi établi l'histoire naturelle des cancers du col avec l'identification des lésions précancéreuses et la relation entre le volume du cancer du sein et la probabilité de dissémination métastatique. Ce domaine de recherche suscite un grand intérêt car il permet d'explorer l'évolution spontanée des cancers, ce que l'on ne pouvait pas étudier chez l'homme puisque dès qu'un cancer es diagnostiqué, il est traité ; ainsi, il donne des bases solides au dépistage des cancers.

Ces exemples montrent les possibilités des recherches épidémiologiques. Les progrès des connaissances sur la biologie des cancers, bien loin de diminuer l'intérêt de ces études, leur ont au contraire donné de nouvelles dimensions d'autant que simultanément les progrès des méthodes statistiques élargissaient leur possibilité. En cette fin de $\mathrm{xx}$ - siècle l'épidémiologie des cancers apparaît plus que jamais comme l'une des branches les plus fécondes de la recherche cancérologique 